\title{
Tunable all-angle negative refraction using antiferromagnets
}

\author{
R. Macêdo ${ }^{1,2}$ and T. Dumelow ${ }^{1, *}$ \\ ${ }^{1}$ Departamento de Física, Universidade do Estado do Rio Grande do Norte, Costa e Silva, 59625-620 Mossoró RN, Brazil \\ ${ }^{2}$ SUPA School of Physics and Astronomy, University of Glasgow, Glasgow G12 8QQ, United Kingdom
}

(Received 18 October 2013; revised manuscript received 7 January 2014; published 22 January 2014; corrected 27 January 2014)

\begin{abstract}
We show how all-angle negative refraction can occur in a uniaxial antiferromagnet close to the magnon resonance frequency. This behavior is based on the fact that, in such cases, the antiferromagnet acts as an indefinite permeability medium, i.e., not all its permeability tensor components are of the same sign. If an external magnetic field is applied, the angle of refraction becomes tunable, and can be made to change sign. We illustrate these effects using the example of $\mathrm{MnF}_{2}$ at low temperature, and show that in this system negative refraction should occur with a large figure of merit.
\end{abstract}

DOI: 10.1103/PhysRevB.89.035135

PACS number(s): 42.25.Bs, 71.36.+c, 78.20.Ls

\section{INTRODUCTION}

When a beam of electromagnetic radiation passes from vacuum (or air) to a negatively refracting medium, it returns to the same side of the surface normal as the incident beam [1]. With a suitable medium, this is possible for all incident angles, both positive and negative, and the resulting effect is referred to as all-angle negative refraction. Although studies of this phenomenon have traditionally concentrated on doublenegative media in which both the permittivity and permeability are negative [2,3], one of the simplest ways of inducing all-angle negative refraction is to make use of anisotropic indefinite media, in which the permittivity or permeability tensor of the refracting medium contains principal components having opposing signs, leading to a hyperbolic dispersion relation $[4,5]$. We stress here that the wave vector and power flow directions are not parallel in anisotropic media. The beam direction is determined by power flow direction, and we define positive or negative refraction accordingly.

Suitable media based on indefinite permittivity may be formed from multilayers [6-11] or aligned parallel nanowire structures [12-15]. In addition, the intrinsic layered structure of graphite has been shown to lead to an indefinite permittivity in the ultraviolet region [16]. At far-infrared frequencies, the phonon response in certain natural anisotropic crystals can also lead to an indefinite permittivity tensor, so that all-angle negative refraction is possible around the optic phonon frequencies in such crystals [17-23].

Phenomena based on hyperbolic dispersion in media with indefinite permeability have received rather less attention. A structure consisting of an array of split ring resonators was reported by Sun et al. [24], and multilayer structures that include natural magnetic materials have also been proposed [25-27]. We should also expect the magnon response in certain natural magnetic crystals to lead to indefinite permeability, in a manner analogous to the way that the phonon response leads to indefinite permittivity in anisotropic crystals such as quartz. In the present paper we consider the case of uniaxial antiferromagnets, confirming that all-angle negative refraction should indeed be possible in such materials. In addition, we show that the angle of refraction should be tunable by applying

\footnotetext{
*Corresponding author: tdumelow@ yahoo.com.br
}

an external magnetic field. We show simulations for the case of $\mathrm{MnF}_{2}$, which, at low temperature, should display such behavior at terahertz frequencies with low losses, corresponding to a large figure of merit.

\section{ALL-ANGLE NEGATIVE REFRACTION IN AN INDEFINITE PERMEABILITY MEDIUM}

The principle of negative refraction in indefinite permittivity media has been discussed in some detail by various authors $[6,7,17,18,28,29]$. This principle is easily adapted to indefinite permeability media, and we summarize the basic principles here. We consider refraction from vacuum to such a medium (in this case to be a uniaxial antiferromagnet) in the geometry shown in Fig. 1. The principal axes of the crystal lie along the Cartesian axes $x, y$, and $z . x z$ is the plane of incidence and $z$ is normal to the slab surface. In particular, we consider the incident radiation to be $s$-polarized (H field in the plane of incidence $x z$ ). The figure includes an external field $\mathbf{B}_{0}$ applied along $y$. Initially we take this field to be zero. In this case the permeability tensor is diagonal:

$$
\boldsymbol{\mu}=\left(\begin{array}{ccc}
\mu_{x x} & 0 & 0 \\
0 & \mu_{y y} & 0 \\
0 & 0 & \mu_{z z}
\end{array}\right) .
$$

If the angle of incidence is represented as $\theta_{1}$, the in-plane wave-vector component $k_{x}$ is given by

$$
k_{x}=k_{0} \sin \theta_{1},
$$

where $k_{0}=\omega / c$. The $z$ component of the incident wave vector is given by

$$
k_{1 z}^{2}=k_{0}^{2}-k_{x}^{2}
$$

and the refracted wave vector by

$$
k_{2 z}^{2}=\varepsilon \mu_{x x} k_{0}^{2}-\frac{\mu_{x x}}{\mu_{z z}} k_{x}^{2},
$$

where $\varepsilon$ represent the dielectric constant (assumed isotropic) of the antiferromagnet. The correct sign of $k_{2 z}$ is determined 


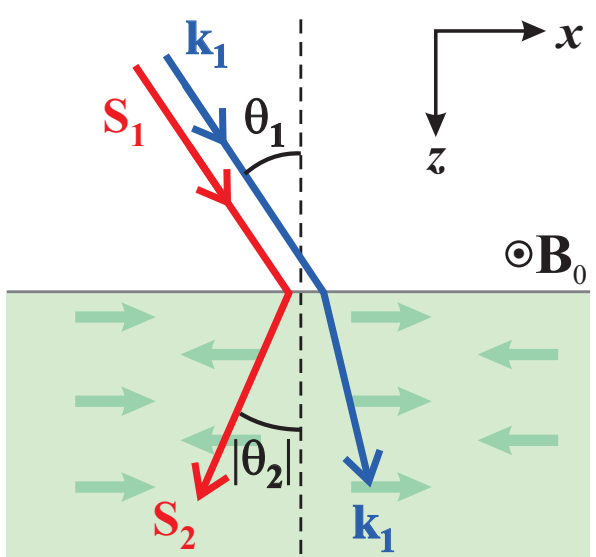

FIG. 1. (Color online) Refraction at the interface between vacuum and an antiferromagnet.

by the condition that power flow must be away from the interface [6].

We consider the angle of refraction $\theta_{2}$ in terms of the direction of the Poynting vector $\mathbf{S}_{2}$ within the antiferromagnet. ${ }^{1}$ In $s$-polarization, the $\mathbf{E}$ field is confined along $y$, so the Poynting vector is most easily represented in terms of the $E_{y}$ field, making use of the conversion $\mathbf{k} \times \mathbf{E}=\omega \mu_{0} \boldsymbol{\mu} \mathbf{H}$. The resulting time-averaged Poynting vector $\left\langle\mathbf{S}_{2}\right\rangle=1 / 2 \operatorname{Re}\left(\mathbf{E} \times \mathbf{H}^{*}\right)$ has components

$$
\begin{aligned}
& \left\langle S_{2 x}\right\rangle=\frac{\left|E_{y}\right|^{2}}{2 \omega \mu_{0}} \operatorname{Re}\left(\frac{k_{x}}{\mu_{z z}}\right), \\
& \left\langle S_{2 y}\right\rangle=0, \\
& \left\langle S_{2 z}\right\rangle=\frac{\left|E_{y}\right|^{2}}{2 \omega \mu_{0}} \operatorname{Re}\left(\frac{k_{2 z}}{\mu_{x x}}\right) .
\end{aligned}
$$

Thus the angle of refraction $\theta_{2}$ is given by

$$
\tan \theta_{2}=\frac{\left\langle S_{2 x}\right\rangle}{\left\langle S_{2 z}\right\rangle}=\frac{\operatorname{Re}\left(k_{x} / \mu_{z z}\right)}{\operatorname{Re}\left(k_{2 z} / \mu_{x x}\right)} .
$$

In order to illustrate the general principle, we initially consider the case when $\mu_{x x}$ and $\mu_{z z}$ are both wholly real, corresponding to zero absorption. Thus $k_{2 z}$ will be either wholly real or wholly imaginary. Of particular interest is the case when $\mu_{x x}>0, \mu_{z z}<0$, which results in a real $k_{2 z}$ [see Eq. (4)]. Since $\left\langle S_{2 z}\right\rangle$ must be positive to ensure power flow into the antiferromagnet, Eq. (5c) shows that $k_{2 z}$ is also positive. A simple comparison of Eqs. (2) and (6) thus shows that $\theta_{1}$ and $\theta_{2}$ have opposing signs, leading to negative refraction.

\section{ALL-ANGLE NEGATIVE REFRACTION IN THE UNIAXIAL ANTIFERROMAGNET $\mathrm{MnF}_{2}$}

In the present paper, we consider the indefinite permeability medium to be a uniaxial antiferromagnet. For such a material,

\footnotetext{
${ }^{1}$ An alternative approach is to consider the direction normal to the equifrequency surface obtained from Eq. (4). The result is essentially the same.
}

the condition $\mu_{x x}>0, \mu_{z z}<0$ may be obtained around the magnon frequencies (typically in the terahertz range) if the antiferromagnet's easy axis lies along $x$. This is the situation shown in Fig. 1. In this case the permeability tensor has components $[30,31]$

$$
\begin{aligned}
& \mu_{x x}=1, \\
& \mu_{y y}=\mu_{z z}=1+\frac{2 \mu_{0} \gamma^{2} B_{A} M_{S}}{\omega_{r}^{2}-(\omega+i \Gamma)^{2}},
\end{aligned}
$$

where $B_{A}$ is the anisotropy field, $M_{S}$ the sublattice magnetization, $\gamma$ the gyromagnetic ratio, and $\Gamma$ a phenomenological damping parameter. The resonance frequency $\omega_{r}$ is given by

$$
\omega_{r}=\gamma\left(2 B_{A} B_{E}+B_{A}^{2}\right)^{1 / 2}
$$

where $B_{E}$ is the exchange field.

In the undamped limit $\Gamma=0$, the condition $\mu_{x x}>0, \mu_{z z}<$ 0 will be satisfied in the frequency interval $\omega_{r}<\omega<\left(\omega_{r}^{2}+\right.$ $\left.2 \mu_{0} \gamma^{2} B_{A} M_{S}\right)^{1 / 2}$. In the more realistic case where $\Gamma \neq 0, \mu_{z z}$ becomes complex, and we can in practice replace the negative refraction condition $\mu_{x x}>0, \mu_{z z}<0$ by $\operatorname{Re}\left(\mu_{x x}\right)>0$, $\operatorname{Re}\left(\mu_{z z}\right)<0$, which occurs over approximately the same frequency interval.

In Fig. 2(a) we show both the real and imaginary parts of $\mu_{z z}$, close to the magnon resonance frequency $\omega_{r}$, for $\mathrm{MnF}_{2}$ at $4.2 \mathrm{~K}$. We show the frequency scale in terms of wave numbers $\omega / 2 \pi c$, as is common for spectroscopic studies. For this material, the relevant parameters are [32] $M_{S}=$ $6.0 \times 10^{5} \mathrm{~A} / \mathrm{m}, B_{A}=0.787 \mathrm{~T}, B_{E}=53.0 \mathrm{~T}$, and $\gamma / 2 \pi c=$ $0.975 \mathrm{~cm}^{-1} / \mathrm{T}$, corresponding to $\omega_{r} / 2 \pi c=8.94 \mathrm{~cm}^{-1}$. The damping parameter is $\Gamma / 2 \pi c=0.0007 \mathrm{~cm}^{-1}$ and the dielectric constant is $\varepsilon=5.5 . \operatorname{Re}\left(\mu_{z z}\right)$ is seen to be negative in the shaded region. Since $\mu_{x x}=1$, negative refraction is expected in this frequency range. Figure 2(b) shows the angle of refraction, calculated using Eq. (6), and confirms that this result for various angles of incidence from vacuum.

We can model refraction of an Gaussian beam obliquely incident on an antiferromagnet using a plane-wave spectrum approach. The incident beam is then represented as a Fourier sum of plane waves:

$$
E_{y}=\int_{-\infty}^{\infty} \psi\left(k_{x}\right) e^{i\left(k_{x} x+k_{1 z} z\right)} d k_{x} .
$$

For a Gaussian beam, $\psi\left(k_{x}\right)$ can be written [33]

$$
\psi\left(k_{x}\right)=-\frac{g}{2 \cos \theta_{1} \sqrt{\pi}} \exp \left[-\frac{g^{2}\left(k_{x}-k_{0} \sin \theta_{1}\right)^{2}}{4 \cos ^{2} \theta_{1}}\right],
$$

where $2 g$ represents the beam width at its waist and $\theta_{1}$ is now an effective incident angle for the overall beam. In practice, we restrict the integral in Eq. (9) to the range $-k_{0} \leqslant k_{x} \leqslant k_{0}$ so that evanescent components, which would be unphysical for the propagating incident beam, are not included [34]. Their contribution to the beam waist would be negligible, so their inclusion would have no practical effect on the refracted or reflected beams, but there would appear large nonzero evanescent fields in the incident beam for $z \ll 0$.

The transmission and reflection of each plane wave component are analyzed separately, and the resulting fields 


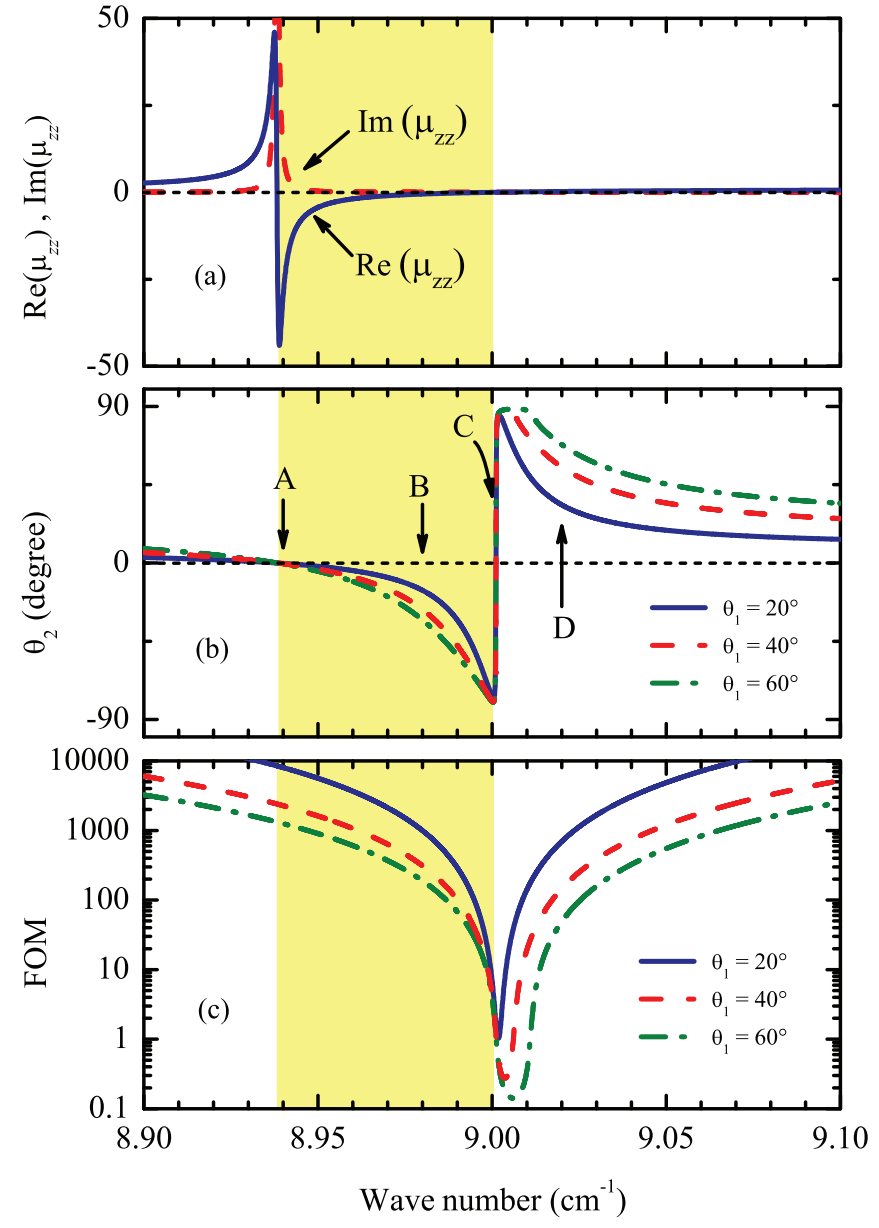

FIG. 2. (Color online) (a) Real and imaginary parts of $\mu_{z z}$ for $\mathrm{MnF}_{2}$ as a function of frequency (expressed as wave number $\omega / 2 \pi c$ ) around the magnon resonance frequency. (b) Angle of refraction $\theta_{2}$ for various angles of incidence from vacuum. (c) Figure of merit for the same angles of incidence. In all figures the yellow shaded region represents the frequency range for which $\operatorname{Re}\left(\mu_{z z}\right)<0$.

summed appropriately to obtain the overall fields, and hence the intensities, at all points in the $x z$ plane [35]. Figure 3 shows the resulting intensities for a Gaussian beam obliquely incident on an $\mathrm{MnF}_{2}$ crystal in the configuration shown in Fig. 1, at the frequencies marked as $A, B, C$, and $D$ on Fig. 2(b). At frequency $A$, corresponding to the magnon resonance frequency $\omega_{r}$, propagation into the antiferromagnet is essentially normal to the surface, as expected from Fig. 2(b). Frequency $B$ is in the negatively refracting frequency range, and Fig. 3(b) confirms this. At frequency $C$, close to the zero in $\operatorname{Re}\left(\mu_{z z}\right)$, the power flow should be nearly parallel to the surface, but, in practice, it is seen that there is very high absorption so there is no effective propagation. Positive refraction is observed at frequency $D$.

All the plots in Fig. 3, except that corresponding to frequency $C$, show propagation into the antiferromagnet with low absorption. The degree of absorption in indefinite (hyperbolic) media is most often represented by a figure of merit (FOM) defined as $[8,11,22,23] \mathrm{FOM}=\left|\operatorname{Re}\left(k_{2 z}\right)\right| / \operatorname{Im}\left(k_{2 z}\right)$. Note that, according to this definition, the FOM is dependent on incident angle. It is plotted in Fig. 2(c) as a function of frequency for the (a)

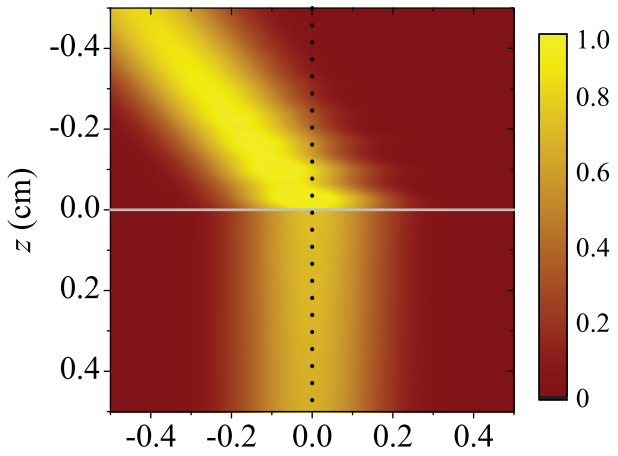

(b)

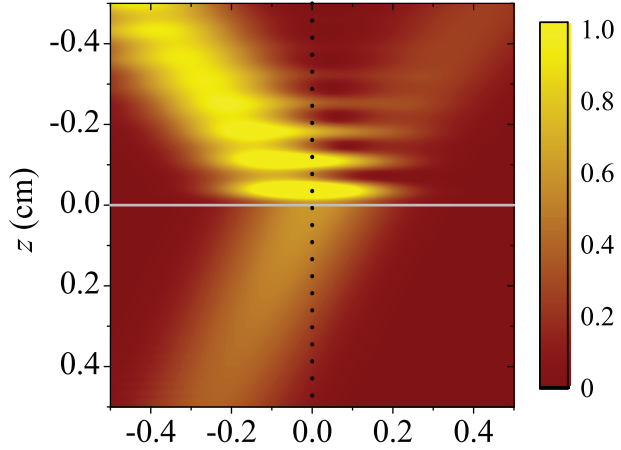

(c)

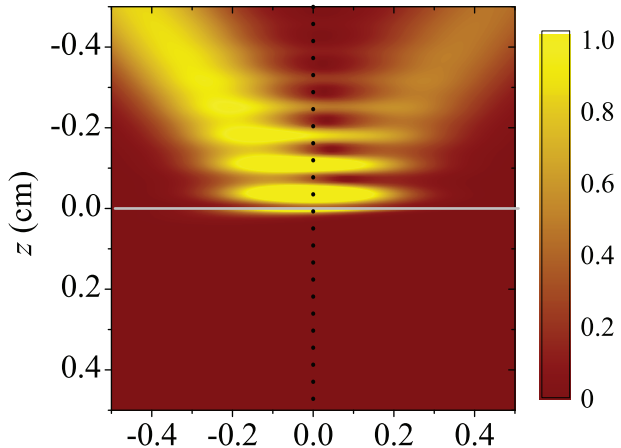

(d)

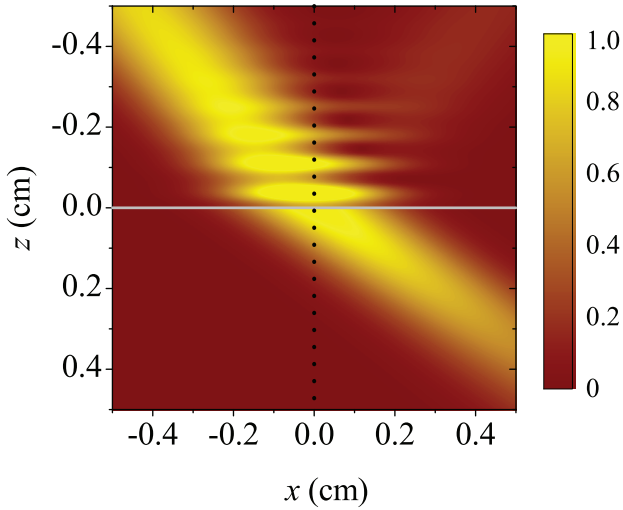

FIG. 3. (Color online) Contour graphs showing intensities (in terms of the magnitude of the time-averaged Poynting vector) for a beam of width $g=0.2 \mathrm{~cm}$ obliquely incident from vacuum, at an angle of incidence of $40^{\circ}$, on an $\mathrm{MnF}_{2}$ crystal at $4.2 \mathrm{~K}$, in the geometry shown in Fig. 1. The antiferromagnet surface is at $z=0$. (a) Frequency $A\left(8.94 \mathrm{~cm}^{-1}\right)$, (b) frequency $B\left(8.98 \mathrm{~cm}^{-1}\right)$, (c) frequency $C\left(9.00 \mathrm{~cm}^{-1}\right)$, (d) frequency $D\left(9.02 \mathrm{~cm}^{-1}\right)$. The vertical dotted lines represent the surface normal.

three incident angles considered in Fig. 2(b). The minimum in the FOM is slightly above frequency $C$, in a narrow frequency region which, in the absence of damping, would correspond to 
$0<\mu_{z z}<(1 / \varepsilon) \sin ^{2} \theta$, with $k_{2 z}$ imaginary and no propagation into the antiferromagnet. In the absence of damping, therefore, the FOM would be zero within this narrow frequency range, and infinite outside it (i.e., in the propagating regions). In the propagating regions, a finite FOM implies absorption. In the case of $\mathrm{MnF}_{2}$, the FOM is large at all frequencies except those close to frequency $C$, in line with the results of Fig. 3. Of particular interest is that, in the negatively refracting region, it several orders of magnitude higher than typically reported for metamaterial structures, so propagating efficiency is high.

\section{EFFECT OF AN EXTERNAL MAGNETIC FIELD ON REFRACTION WITHIN THE ANTIFERROMAGNET}

The above account describes negative refraction in antiferromagnets in the absence of any external field. We now consider the effect of applying a static nonzero magnetic field $\mathbf{B}_{0}$ is applied along $y$, perpendicular to the uniaxis (see Fig. 1), noting that, in this geometry, any effects involving mixing between $s$ - and $p$-polarizations are avoided.

In the presence of such a field, the spins will become canted by an angle $\alpha$ given by

$$
\sin \alpha=\frac{B_{0}}{B_{A}+2 B_{E}} .
$$

The main effect of this canting is to raise the resonance frequency $\omega_{r}$ that appears in $\mu_{z z}$ to a higher value $\omega_{\perp}$ given by $[31,36]$

$$
\omega_{\perp}=\left(\omega_{r}^{2} \cos ^{2} \alpha+2 \gamma^{2} B_{0} \sin \alpha\right)^{1 / 2} .
$$

$\mu_{z z}$ is then given by

$$
\mu_{z z}=1+\frac{2 \mu_{0} \gamma^{2} M_{S}\left(B_{0} \sin \alpha+B_{A} \cos 2 \alpha\right)}{\omega_{\perp}^{2}-(\omega+i \Gamma)^{2}} .
$$

The resulting $\operatorname{Re}\left(\mu_{z z}\right)$ values for $\mathrm{MnF}_{2}$ are shown in Fig. 4 for various values of applied field $B_{0}$.

Although the main effect of the external field is simply to shift the features in $\mu_{z z}$ to higher frequency, the other components of the permeability tensor are also slightly affected, and the off-diagonal components $\mu_{x z}$ and $\mu_{z x}$ become

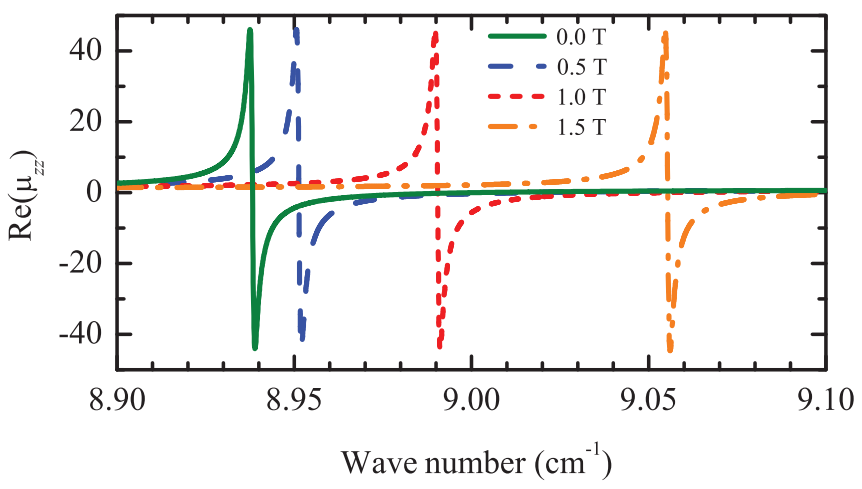

FIG. 4. (Color online) $\operatorname{Re}\left(\mu_{z z}\right)$ plots for $\mathrm{MnF}_{2}$ for various values of applied field $B_{0}$.

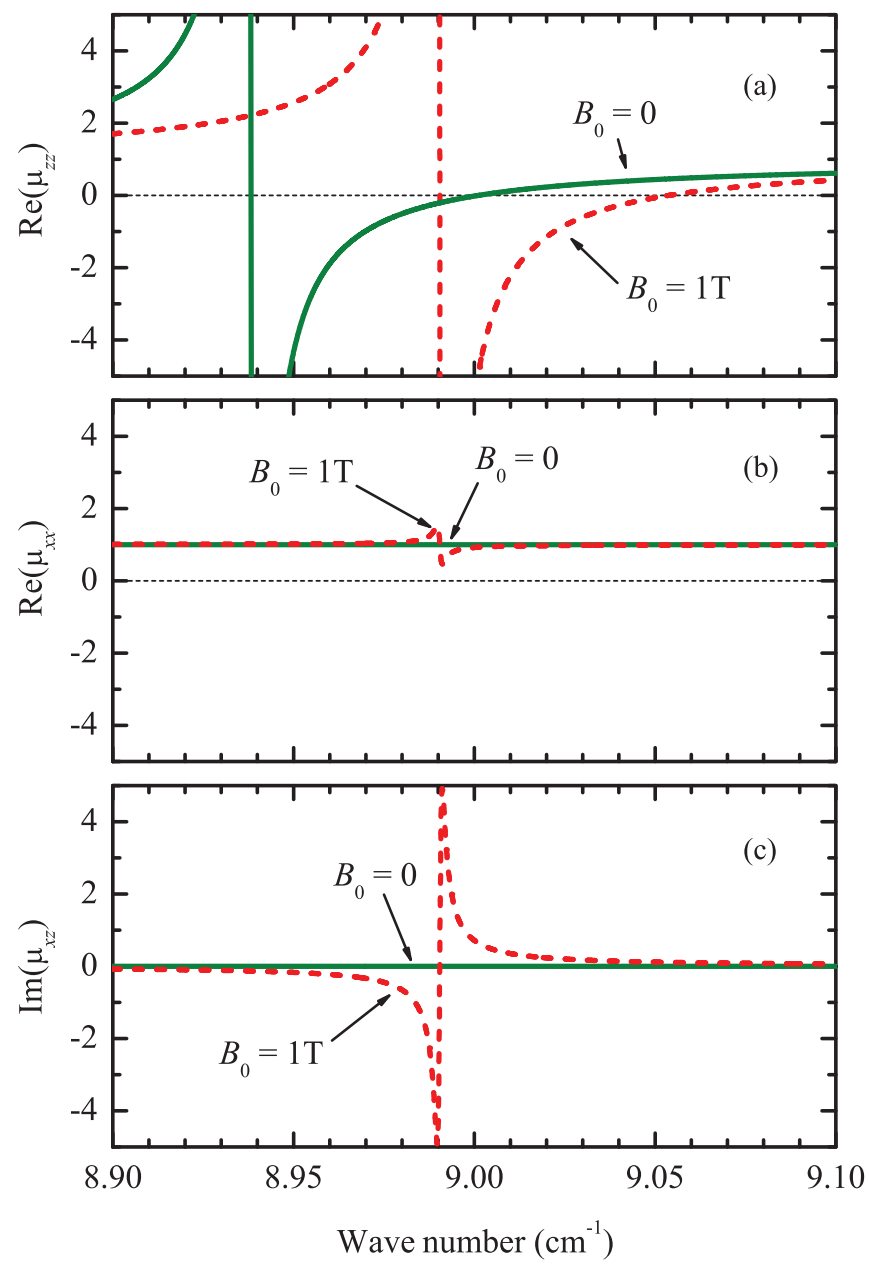

FIG. 5. (Color online) (a) $\operatorname{Re}\left(\mu_{z z}\right)$, (b) $\operatorname{Re}\left(\mu_{x x}\right)$, and (c) $\operatorname{Im}\left(\mu_{x z}\right)$ for $B_{0}=0$ and $B_{0}=1 \mathrm{~T}$.

nonzero [36]. The components other than $\mu_{z z}$ that contribute in $s$-polarization are $[31,36]$

$$
\begin{aligned}
& \mu_{x x}=1+\frac{2 \mu_{0} \gamma^{2} B_{0} M_{S} \sin \alpha}{\omega_{\perp}^{2}-(\omega+i \Gamma)^{2}}, \\
& \mu_{x z}=-\mu_{z x}=-i \frac{2 \mu_{0} \gamma M_{S}(\omega+i \Gamma) \sin \alpha}{\omega_{\perp}^{2}-(\omega+i \Gamma)^{2}} .
\end{aligned}
$$

The values of $\operatorname{Re}\left(\mu_{x x}\right)$ and $\operatorname{Im}\left(\mu_{x z}\right)$ are compared with $\operatorname{Re}\left(\mu_{z z}\right)$ in Fig. 5, on an expanded scale, with and without an applied field (in order to simplify the figure, only one nonzero value of the applied field, corresponding to $B_{0}=1 \mathrm{~T}$, is shown). Note that we show $\operatorname{Im}\left(\mu_{x z}\right)$ rather than $\operatorname{Re}\left(\mu_{x z}\right)$ because it is the imaginary component that shows up the characteristic resonant structure in the case of $\mu_{x z}$ (the real part consists of a weak peak at $\omega_{\perp}$ ). For $B_{0} \neq 0$, all plots show resonances at $\omega_{\perp}$, but the resonances in $\mu_{x x}$ and $\mu_{x z}$ are much weaker than that in $\mu_{z z}$ since the canting angle is small $\left(B_{0}=1 \mathrm{~T}\right.$ corresponds to a canting angle of $\alpha=0.54^{\circ}$ ).

In order to study refraction we once again look at the Poynting vector. Explicit $s$-polarization calculation of the 


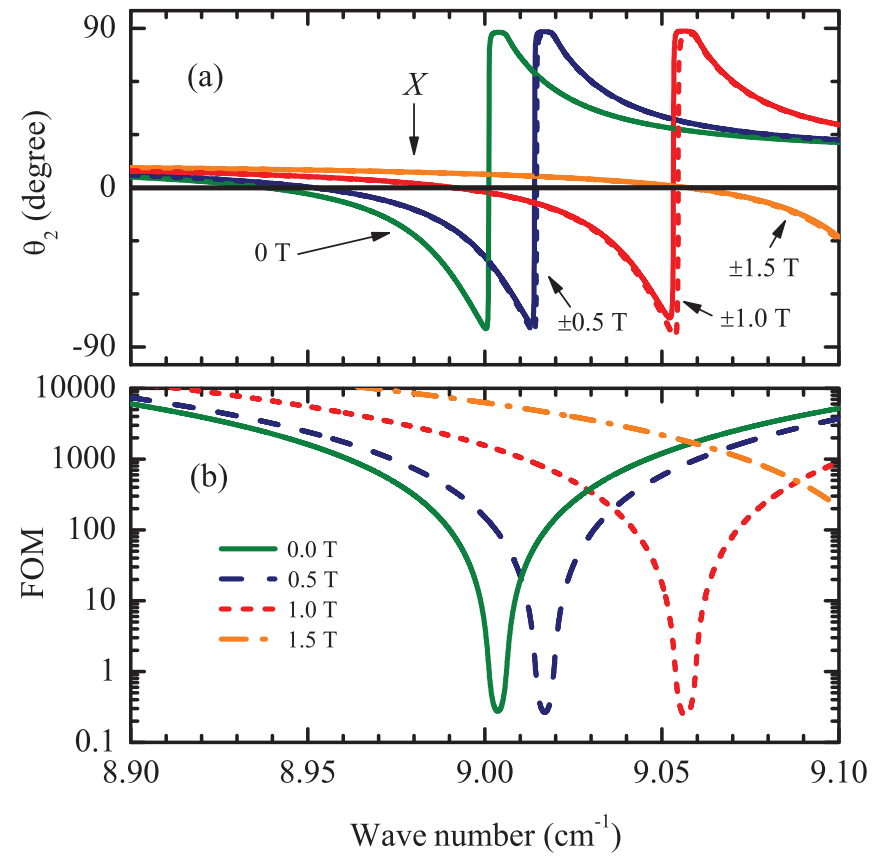

FIG. 6. (Color online) (a) Angle of refraction $\theta_{2}$ in $\mathrm{MnF}_{2}$ for various values of applied field $B_{0}$, for an incident angle of $\theta_{1}=40^{\circ}$. (b) Figure of merit for the same angle of incidence and applied fields. In part (a) the solid lines represent positive fields and the dashed lines represent negative fields.

Poynting vector components in the configuration of Fig. 1 gives

$$
\begin{aligned}
\left\langle S_{2 x}\right\rangle & =\frac{\left|E_{y}\right|^{2}}{2 \omega \mu_{0}} \operatorname{Re}\left(\frac{k_{x} \mu_{x x}+k_{2 z} \mu_{x z}}{\mu_{x x} \mu_{z z}+\mu_{x z}^{2}}\right), \\
\left\langle S_{2 y}\right\rangle & =0, \\
\left\langle S_{2 z}\right\rangle & =\frac{\left|E_{y}\right|^{2}}{2 \omega \mu_{0}} \operatorname{Re}\left(\frac{k_{2 z} \mu_{z z}-k_{x} \mu_{x z}}{\mu_{x x} \mu_{z z}+\mu_{x z}^{2}}\right),
\end{aligned}
$$

where the wave-vector component $k_{2 z}$ is now obtained from

$$
k_{2 z}^{2}=\frac{\varepsilon k_{0}^{2}\left(\mu_{x x} \mu_{z z}+\mu_{x z}^{2}\right)-\mu_{x x} k_{x}^{2}}{\mu_{z z}} .
$$

The angle of refraction may once more be calculated using $\tan \theta_{2}=\left\langle S_{2 x}\right\rangle /\left\langle S_{2 z}\right\rangle$. The resulting values, assuming an incident angle of $\theta_{1}=40^{\circ}$, are shown in Fig. 6(a) for a series of both positive and negative applied fields (positive fields being directed along $+y$ and negative fields along $-y$ ). The corresponding FOMs are shown in Fig. 6(b). Both sets of curves are effectively shifted to higher frequency by the application of an external field, but are otherwise unchanged to any significant degree, despite the increased complexity that might be expected from the new features that appear in $\mu_{x x}$ and $\mu_{x z}$. Thus the refracting behavior is very similar to that discussed in the previous section (i.e., for $B_{0}=0$ ), and, in addition, the application of an applied field does not adversely affect the FOM to any noticeable extent.

In analyzing the effect of reversing the direction of $\mathbf{B}_{0}$ [in effect, changing the sign of $\mu_{x z}$, and hence $\mu_{z x}$; see Eq. (14b)],

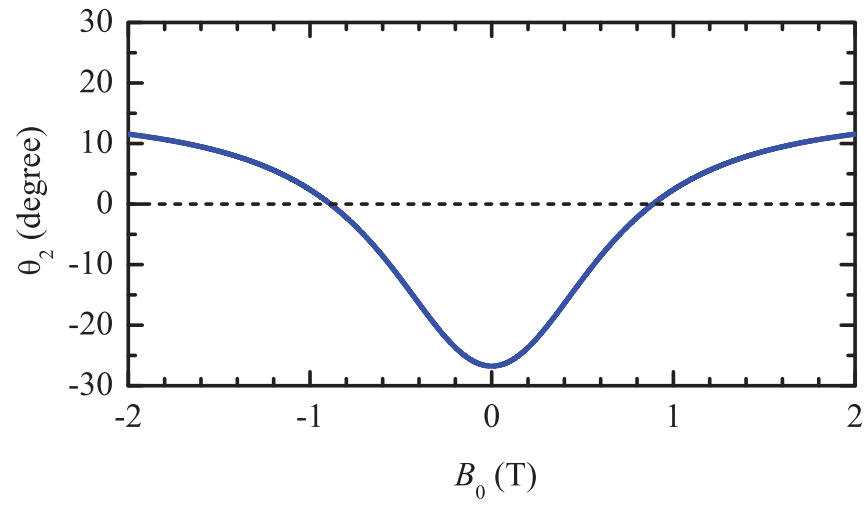

FIG. 7. (Color online) Angle of refraction $\theta_{2}$ in $\mathrm{MnF}_{2}$ as a function of applied field $B_{0}$ at frequency $X\left(8.98 \mathrm{~cm}^{-1}\right)$.

we note that, in the limit of zero damping (i.e., zero absorption), $\mu_{x x}$ and $\mu_{z z}$ would both be real and $\mu_{x z}$ would be imaginary. As in the zero field case, $k_{2 z}$ would be either wholly real or wholly imaginary, being real if there were propagation within the antiferromagnet and imaginary where there is not (corresponding to a narrow frequency region just above the zero in $\mu_{z z}$ ). In the former case, which is that of interest when considering refraction, it is straightforward to see from Eqs. (15) that none of the Poynting vector components would depend on the sign of $\mu_{x z}$. The angle of refraction $\theta_{2}$ would therefore be independent of the sign of $B_{0}$, and we would say that it is reciprocal with respect to field reversal. In practice, therefore, any nonreciprocal effects in the power flow direction are associated with the imaginary part of $k_{2 z}$. At frequencies at which propagation is expected, the imaginary part is due to absorption.

The above analysis is in agreement with the results in Fig. 6(a). For most of the spectrum, reversing the sign of $B_{0}$ has negligible effect. However, near the relevant minimum in the FOM (close to the zero in $\mu_{z z}$ ), at which there is a significant imaginary contribution to $k_{2 z}$, some nonreciprocity is seen. Nevertheless, the behavior at such frequencies is similar to that shown in Fig. 3(b), and it is not realistic to speak of refraction in the normal sense. In the regions in which it makes sense to discuss refraction, positive or negative, it is reasonable to say that refraction is reciprocal. Any nonreciprocal behavior present is simply due to absorption. This is similar to the type of nonreciprocity observed in the intensity of the reflected beam. Nonreciprocity in this intensity also only exists in the presence of absorption [31,37-40].

We now look at how the angle of refraction can be tuned by varying the applied magnetic field. Since the $\theta_{2}$ curves in Fig. 6(a) depend on $B_{0}$, at any given frequency the angle of refraction will depend on the magnitude of the applied field. We consider the frequency marked as $X\left(8.98 \mathrm{~cm}^{-1}\right)$ in Fig. 6(a). As can be seen from the figure, at this frequency the angle of refraction should vary from negative to positive as the applied field is increased. We can see this in Fig. 7, which shows this behavior for both positive and negative fields and confirms the reciprocal nature of the angle of refraction.

We can verify the predicted behavior by studying that of a Gaussian beam incident on the surface of the antiferromagnet for various values of applied field, using the same type of 
(a)

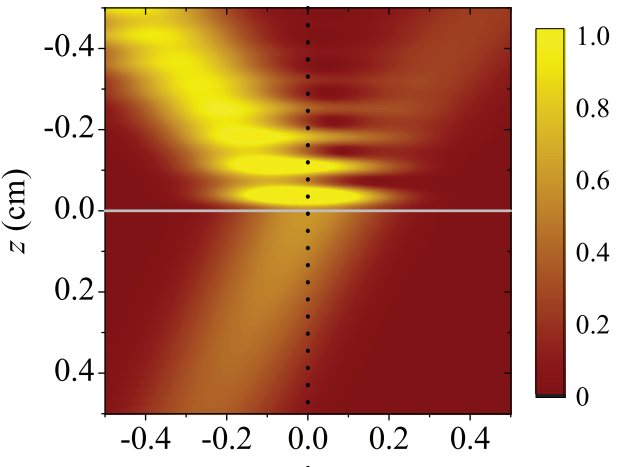

(b)

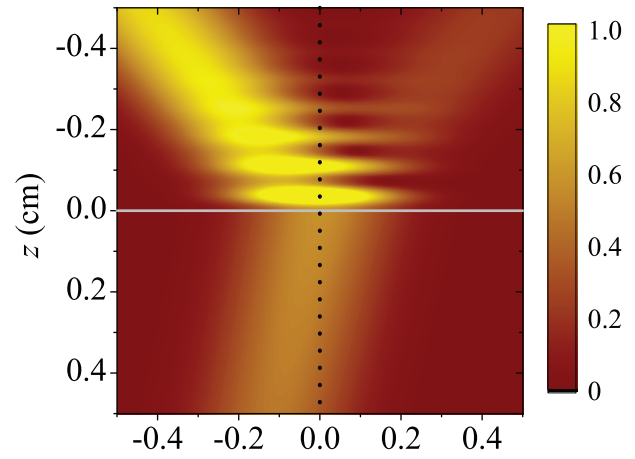

(c)

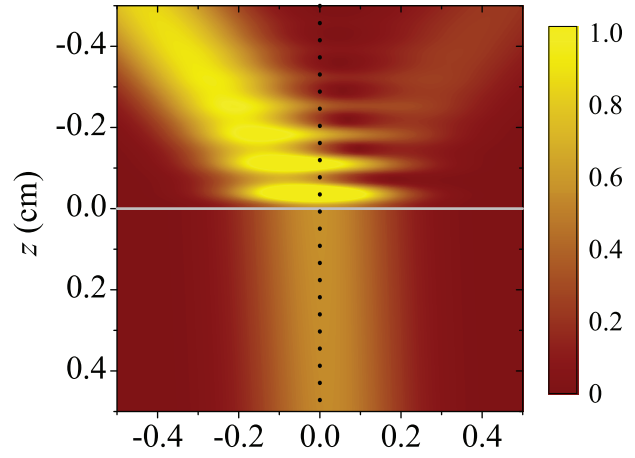

(d)

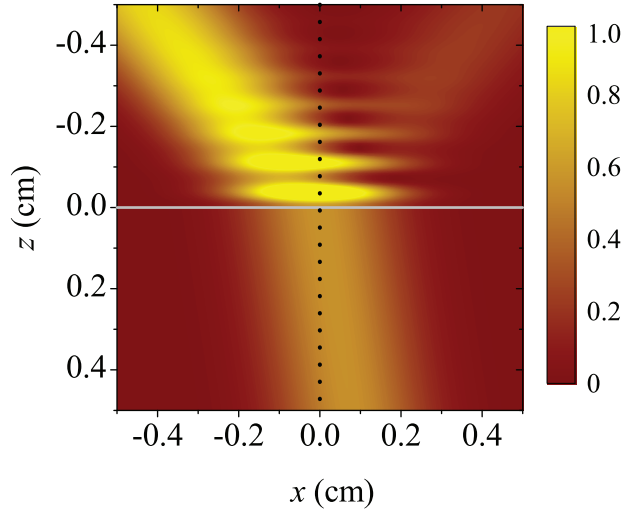

FIG. 8. (Color online) Contour graphs showing intensities for a beam of width $g=0.2 \mathrm{~cm}$ obliquely incident from vacuum, at an angle of incidence of $40^{\circ}$, on an $\mathrm{MnF}_{2}$ crystal at frequency $X$ $\left(8.98 \mathrm{~cm}^{-1}\right)$, in the presence of an external field $\mathbf{B}_{0}$. (a) $B_{0}=0$, (b) $B_{0}=0.5 \mathrm{~T}$, (c) $B_{0}=1 \mathrm{~T}$, (d) $B_{0}=1.5 \mathrm{~T}$.

analysis used in obtaining Fig. 3. The results for various positive values of $B_{0}$ are shown in Fig. 8 (results for negative $B_{0}$ are essentially the same, as expected from Fig. 7). Figure 8 confirms that the angle of refraction can be tuned from negative to positive using an applied external magnetic field, and that, in the case of the chosen frequency $X$ (for which the FOM is high for all values of $B_{0}$ ), propagation into the antiferromagnet occurs without significant absorption.

\section{DISCUSSION}

The above analysis suggests that tunable negative refraction based on antiferromagnets should be feasible. We have considered a geometry in which the applied field is perpendicular to the antiferromagnet's easy axis, which, in turn, lies in the plane of incidence. The present result contrasts with that for a previously studied geometry in which the easy axis lies parallel to the applied field (i.e., along $y$ ) [41,42], In such a case, refraction of a strictly propagating beam is always positive, power flow being parallel to the wave vector even in a nonzero field.

Propagating efficiency, as represented by the figure of merit, is very high in this system. This is clear from the results shown in Fig. 8, which shows no visible decay on propagation through the antiferromagnet. Low temperature is necessary to achieve such efficiency, however. We note that high propagation efficiency has also been predicted in certain natural indefinite permittivity materials at liquidhelium temperatures. In particular, it has been pointed out that the phonon response in triglycine sulfate (TGS) [17] and the plasma response in bismuth [43] should both lead to indefinite permittivity with low absorption.

The fact that the angle of refraction is reciprocal with respect to field reversal is important in that it implies that changing the sign of the angle of incidence simply changes the sign of the angle of refraction [i.e., $\theta_{2}\left(\theta_{1}\right)=-\theta_{2}\left(-\theta_{1}\right)$ ]. Thus, even in the presence of an external field in the configuration of Fig. 1, a single antiferromagnet crystal with parallel sides should act as a slab lens similar to that obtained from an indefinite permittivity medium $[7,15,17,22]$. An object placed on one side of such a lens will project an intermediate image within the slab and a final image on the other side of it. In general, these images will not be perfect, and the crystal will not have the superlensing properties of double-negative slab lenses discussed by Pendry [3]. Nevertheless, a slab lens of tunable "focal length" (i.e., tunable object-image distance) should in principle be possible.

An important property of hyperbolic media is that they can convert evanescent waves in vacuum into propagating waves within the medium. In terms of a slab lens, this is significant in the case where the material parameters are such that both the object and image can be very close to (i.e., within the near field of) the crystal surfaces, since in this case subwavelength imaging should be possible. We would expect this behavior close to the resonance frequency $\omega_{\perp}$ [22], in which case propagation across the crystal would be in the form of nearly collimated beams [44]. Thus, both the more general possibility of a slab lens with tunable "focal length" and that of obtaining subwavelength imaging close to $\omega_{\perp}$ warrant further investigation.

\section{CONCLUSIONS}

We have shown that a uniaxial antiferromagnet such as $\mathrm{MnF}_{2}$ should act as a negatively refracting medium due to its indefinite permeability. Furthermore, the refracting angle can be tuned by the application of an external magnetic field. In 
the case of this particular material, losses are expected to be very low, and the refracted beam should propagate with high efficiency. This type of refraction has potential for use in slab lenses with tunable properties.

\section{ACKNOWLEDGMENT}

The work was partially financed by the Brazilian research agency $\mathrm{CNPq}$.
[1] S. A. Ramakrishna, Rep. Prog. Phys. 68, 449 (2005).

[2] V. G. Veselago, Sov. Phys. Usp. 10, 509 (1968).

[3] J. B. Pendry, Phys. Rev. Lett. 85, 3966 (2000).

[4] I. V. Lindell, S. A. Tretyakov, K. I. Nikoskinen, and S. Ilvonen, Microwave Opt. Technol. Lett. 31, 129 (2001).

[5] D. R. Smith, P. Kolinko, and D. Schurig, J. Opt. Soc. Am. B 21, 1032 (2004).

[6] T. Dumelow and D. R. Tilley, J. Opt. Soc. Am. A 10, 633 (1993).

[7] L. V. Alekseyev and E. Narimanov, Opt. Express 14, 11184 (2006).

[8] A. J. Hoffman, L. Alekseyev, S. S. Howard, K. J. Franz, D. Wasserman, V. A. Podolskiy, E. E. Narimanov, D. L. Sivco, and C. Gmachl, Nat. Mater. 6, 946 (2007).

[9] M. Scalora, G. D’Aguanno, N. Mattiucci, M. J. Bloemer, D. de Ceglia, M. Centini, A. Mandatori, C. Sibilia, N. Akozbek, M. G. Cappeddu, M. Fowler, and J. W. Haus, Opt. Express 15, 508 (2007).

[10] M. A. K. Othman, C. Guclu, and F. Capolino, Opt. Express 21, 7614 (2013).

[11] M. A. K. Othman, C. Guclu, and F. Capolino, J. Nanophotonics 7, 073089 (2013).

[12] J. Yao, Z. Liu, Y. Liu, Y. Wang, C. Sun, G. Bartal, A. M. Stacy, and X. Zhang, Science 321, 930 (2008).

[13] W. T. Lu and S. Sridhar, Phys. Rev. B 77, 233101 (2008).

[14] Y. Liu, G. Bartal, and X. Zhang, Opt. Express 16, 15439 (2008).

[15] J. Yao, K.-T. Tsai, Y. Wang, Z. Liu, G. Bartal, Y.-L. Wang, and X. Zhang, Opt. Express 17, 22380 (2009).

[16] J. Sun, J. Zhou, B. Li, and F. Kang, Appl. Phys. Lett. 98, 101901 (2011).

[17] T. Dumelow, J. A. P. da Costa, and V. N. Freire, Phys. Rev. B 72, 235115 (2005).

[18] V. Dvorak and P. Kuzel, Ferroelectrics 338, 195 (2006).

[19] O. S. Eritsyan, A. A. Lalayan, O. M. Arakelyan, A. A. Papoyan, and R. B. Kostanyan, Crystallogr. Rep. 55, 938 (2010).

[20] R. Wang, J. Sun, and J. Zhou, Appl. Phys. Lett. 97, 031912 (2010).

[21] R. Rodrigues da Silva, R. Macêdo da Silva, T. Dumelow, J. A. P. da Costa, S. B. Honorato, and A. P. Ayala, Phys. Rev. Lett. 105, 163903 (2010).
[22] R. Estevâm da Silva, R. Macêdo, T. Dumelow, J. A. P. da Costa, S. B. Honorato, and A. P. Ayala, Phys. Rev. B 86, 155152 (2012).

[23] R. Macêdo, R. Rodrigues da Silva, T. Dumelow, and J. A. P. da Costa, Opt. Commun. 310, 94 (2014).

[24] J. Sun, L. Kang, R. Wang, L. Liu, L. Sun, and J. Zhou, New J. Phys. 12, 083020 (2010).

[25] R. Tarkhanyan and D. Niarchos, J. Magn. Magn. Mater. 312, 6 (2007).

[26] R. Tarkhanyan, D. Niarchos, and M. Kafesaki, J. Magn. Magn. Mater. 322, 603 (2010).

[27] W. Li, Z. Liu, X. Zhang, and X. Jiang, Appl. Phys. Lett. 100, 161108 (2012).

[28] O. S. Eritsyan, Kristallografiya 23, 461 (1978).

[29] P. A. Belov, Microwave Opt. Technol. Lett. 37, 259 (2003).

[30] D. L. Mills and E. Burstein, Rep. Prog. Phys. 37, 817 (1974).

[31] K. Abraha and D. R. Tilley, Surf. Sci. Rep. 24, 129 (1996).

[32] T. Dumelow and M. C. Oliveros, Phys. Rev. B 55, 994 (1997).

[33] B. R. Horowitz and T. Tamir, J. Opt. Soc. Am. 61, 586 (1971).

[34] X. Chen and C.-F. Li, Phys. Rev. E 69, 066617 (2004).

[35] J. A. Kong, B.-I. Wu, and Y. Zhang, Appl. Phys. Lett. 80, 2084 (2002).

[36] N. S. Almeida and D. L. Mills, Phys. Rev. B 37, 3400 (1988).

[37] L. Remer, B. Lüthi, H. Sauer, R. Geick, and R. E. Camley, Phys. Rev. Lett. 56, 2752 (1986).

[38] R. E. Camley, Surf. Sci. Rep. 7, 103 (1987).

[39] R. L. Stamps, B. L. Johnson, and R. E. Camley, Phys. Rev. B 43, 3626 (1991).

[40] D. E. Brown, T. Dumelow, T. J. Parker, K. Abraha, and D. R. Tilley, Phys. Rev. B 49, 12266 (1994).

[41] T. Dumelow, J. A. P. da Costa, F. Lima, and E. L. Albuquerque, in Recent Optical and Photonic Technologies, edited by K. Y. Kim (In-Tech, Vukovar, 2010), pp. 143-168, Chap. 8.

[42] F. Lima, T. Dumelow, E. L. Albuquerque, and J. A. P. da Costa, J. Opt. Soc. Am. B 28, 306 (2011).

[43] V. A. Podolskiy, L. V. Alekseyev, and E. E. Narimanov, J. Mod. Opt. 52, 2343 (2005).

[44] P. A. Belov, C. R. Simovski, and P. Ikonen, Phys. Rev. B 71, 193105 (2005). 